Joseph T. Flynn • Stephen J. Warnick

\title{
Isradipine treatment of hypertension in children: a single-center experience
}

Received: 11 April 2002 / Revised: 31 May 2002 / Accepted: 4 June 2002 / Published online: 2 August 2002 (C) IPNA 2002

\begin{abstract}
Many children with hypertension, particularly those with new-onset hypertension related to glomerulonephritis, organ transplantation, or other forms of secondary hypertension, require treatment with a shortacting antihypertensive in order to quickly achieve blood pressure (BP) control. We administered isradipine, a short-acting, second-generation calcium antagonist, to 72 such children. Retrospective data collection was undertaken to determine the effects of isradipine treatment. The mean age of children treated with isradipine was $74 \pm 55$ months (mean \pm SD). Nearly all of these children had secondary hypertension and were initially treated as hospital inpatients for newly diagnosed hypertension. Mean isradipine dose was $0.36 \pm 0.17 \mathrm{mg} / \mathrm{kg}$ per day, with no significant variation in dose according to patient age. Isradipine was administered three times per day in most instances, but $21 \%$ of the time it was administered four times per day. An extemporaneous isradipine suspension was used in $62 \%$ of treatment courses. BP control was achieved with isradipine alone in 38 children; the remainder received isradipine in combination with additional antihypertensives. Comparison of pre-treatment BP with BP obtained $8 \pm 9$ days later demonstrated a significant $\mathrm{BP}$ reduction with isradipine treatment, with a mean reduction of $14 \pm 13 \mathrm{mmHg}$ for systolic $\mathrm{BP}$ and $13 \pm 15 \mathrm{mmHg}$ for diastolic BP. There was no effect of isradipine treatment on heart rate. Adverse effects occurred in $9.5 \%$ of treatment courses, and included headache, flushing, dizziness, and tachycardia. We conclude
\end{abstract}

\section{J.T. Flynn (}

Department of Pediatrics, Division of Pediatric Nephrology,

Albert Einstein College of Medicine, Bronx, New York, USA e-mail: jflynn@montefiore.org

Tel.: +1-718-6551120, Fax: +1-718-6523136

\section{S.J. Warnick}

Undergraduate Research Opportunity Program,

College of Literature, Science and the Arts,

University of Michigan, Ann Arbor, Michigan, USA

\section{J.T. Flynn}

Division of Pediatric Nephrology, Montefiore Medical Center,

111 East 210th Street, Bronx, NY 10467, USA that isradipine successfully lowers BP in hypertensive children with secondary forms of hypertension. Use of isradipine suspension allows infants and young children to be treated as readily as older children.

Keywords Clinical trial $\cdot$ Calcium channel blocker . Dihydropyridine $\cdot$ Secondary hypertension $\cdot$ Nifedipine . Amlodipine

\section{Introduction}

Treatment of hypertensive children has been hampered by the limited information available regarding efficacy and safety of antihypertensive medications in children [1]. This problem is perhaps most significant for more recently introduced calcium antagonists and angiotensin converting enzyme inhibitors, which have been recommended as first-line agents for use in hypertensive children [2]. Additionally, few antihypertensive agents are available in formulations suitable for administration to infants and toddlers, which means that suspensions must be compounded locally, many of which may not be uniform in potency or stable for long periods of time $[1,3]$.

Treatment of children with hypertension of acute onset (such as that caused by acute glomerulonephritis, organ transplantation, or other conditions) is further compounded by the lack of an agent that could achieve blood pressure (BP) control relatively rapidly, within a few days at most, with a low incidence of adverse effects. Ease of administration and of titration would be additional desirable attributes of such an agent. Many currently available antihypertensive agents, however, do not fulfill these criteria. Vasodilators such as hydralazine and minoxidil have significant adverse effects or cannot be compounded into stable suspensions [4, 5]. Betaadrenergic antagonists (i.e., propranolol or labetalol) may cause bradycardia or bronchospasm [6]. First-generation calcium antagonists either have adverse cardiovascular effects (verapamil) or are difficult to administer 
(nifedipine), whereas the third-generation calcium antagonist amlodipine may not achieve therapeutic effect rapidly enough [7]. Angiotensin converting enzyme inhibitors may be contraindicated because of transient renal dysfunction.

Isradipine, a second-generation dihydropyridine calcium antagonist, has several characteristics that would appear to make it an ideal agent for treatment of hypertension of acute onset. It has high affinity for L-type calcium channels located in vascular smooth muscle, and little effect on myocardial calcium channels, leading to a fairly specific and potent antihypertensive effect [8]. Onset of action is rapid, within $30 \mathrm{~min}$ of oral administration, a property that has led some investigators to utilize isradipine as a treatment for urgent hypertension [9]. When used in chronic treatment of hypertension in adults, isradipine is generally well tolerated, with a favorable adverse effect profile compared with the firstgeneration calcium antagonist nifedipine $[10,11]$. Finally, and perhaps most importantly as far as treatment of children is concerned, isradipine may be easily compounded into a stable extemporaneous suspension [12]. For these reasons, isradipine was applied to the treatment of hypertensive children at the University of Michigan in 1994. Our experience with the use of this agent in children is summarized in this report.

\section{Materials and methods}

\section{Patient eligibility}

Patients were eligible for inclusion in this study if they had received treatment for hypertension (as defined below) with isradipine between July 1995 and May 2000. Pediatric nephrology fellows and attending physicians identified potential patients to the principal investigator, who then reviewed their medical records to confirm eligibility. The investigators' database was cross-referenced with dispensing records from the medical center's outpatient pharmacy in order to identify additional children that had been treated with isradipine at the institution during the study period. All children so identified were then added to the investigators' database.

\section{Data collection}

Data for this retrospective study were collected by review of patients' medical records under the auspices of an ongoing antihypertensive database maintained by the Division of Pediatric Nephrology. The University of Michigan Institutional Review Board for Medical Research granted approval for maintenance of this database on an annual basis.

Data were collected utilizing a standardized collection form as previously described [13]. All data collection was performed by a single individual (S.W.) who had been trained by the principal investigator (J.F.). Quality control of the data collection was maintained by ongoing review of the completed data collection forms by the principal investigator.

Demographic information collected included patient's age, weight, underlying diagnosis, presence or absence of hypertension prior to isradipine use, and treatment with other antihypertensive medications prior to use of isradipine. Vital signs, including systolic BP, diastolic BP, and heart rate were recorded from inpatient nursing flow sheets and outpatient clinic records. Isradipine dose and formulation, use of concomitant antihypertensive medications, and adverse effects reported by patients were also recorded.
To determine the effects of isradipine treatment in hospitalized patients, the patients' $\mathrm{BP}$ and heart rate during the $24 \mathrm{~h}$ prior to the use of isradipine were recorded, followed by their BP, heart rate, and isradipine dose on the day of discharge from the hospital. For outpatients, clinic BP and heart rates were recorded for the clinic visit immediately preceding the initial use of isradipine, followed by the patients' $\mathrm{BP}$, heart rates, and isradipine dose on the first follow-up visit.

\section{BP measurement and definitions}

BP in all patients was measured by either the hospital inpatient nursing staff or outpatient clinic staff using automated oscillometric BP monitors (Dynamap, Critikon, Tampa, Fla., USA). In hospitalized patients, BP was measured every $1-4 \mathrm{~h}$ as warranted by the patient's clinical condition. Clinic BP was measured in the right arm with the child seated; choice of extremity and patient position in hospitalized patients varied according to their clinical condition.

Hypertension in all patients was defined as a systolic and/or diastolic BP in excess of the 95th percentile for that child's age, gender, and height [2]. Target BP for all patients treated with isradipine was set at the 90th percentile [14].

\section{Isradipine treatment}

Children eligible for treatment with isradipine included any child with hypertension who could be treated with an oral agent. Isradipine was generally not used for the treatment of urgent hypertension; at our center, such children were typically treated with intravenous labetalol as previously described [7]. However, children receiving ongoing chronic treatment with isradipine could receive additional doses for acute BP elevations if necessary.

Isradipine was started at a dose of $0.05-0.1 \mathrm{mg} / \mathrm{kg}$ per dose every $8 \mathrm{~h}$ and adjusted as necessary to reduce the patient's BP, until the target BP was reached. Frequency of dosing was increased to every $6 \mathrm{~h}$ if the patient's BP frequently exceeded the target range more than $1 \mathrm{~h}$ before the next scheduled isradipine dose, or if additional doses were frequently being administered as described above. If BP control was not achieved with isradipine alone, additional antihypertensive agents were added as necessary.

Isradipine was administered either as commercially available 2.5- or 5-mg immediate-release capsules (DynaCirc, Novartis Pharmaceuticals, East Hanover, N.J., USA) or as a $1.0 \mathrm{mg} / \mathrm{ml} \mathrm{ex-}$ temporaneous suspension compounded by the C.S. Mott Children's Hospital pharmacy [12]. Occasional patients who were receiving a total daily dose of isradipine of $5 \mathrm{or} 10 \mathrm{mg}$ were switched to commercially available extended-release tablets (DynaCirc CR, Novartis Pharmaceuticals) administered once or twice daily.

\section{Data analysis}

Database software used included the Medlog clinical data management system (Information Analysis Corporation, Incline Village, Nev., USA) and Excel 97 (Microsoft Corporation, Redmond, Wash., USA). Further data analysis was performed utilizing SPSS for Windows 10.0.5 (SPSS, Chicago, Ill., USA).

Except where otherwise noted, data included in this report are expressed as mean \pm standard deviation, or as percentages. Statistical tests utilized included two-tailed, paired and unpaired $t$-tests to examine differences in group means for normally distributed variables and the Mann-Whitney and Wilcoxon signed ranks tests for non-normally distributed variables. The Chi-square test or Fisher's exact test was used to examine the differences in proportions between groups. Linear regression analysis was performed to examine the relationship between variables. Statistical significance for all analyses was set at $P<0.05$. 
Table 1 Comparison of patients receiving isradipine monotherapy with those receiving combination therapy $(B P$ blood pressure)

\begin{tabular}{lccc}
\hline Characteristic & $\begin{array}{l}\text { Isradipine monotherapy } \\
(n=38)\end{array}$ & $\begin{array}{l}\text { Combination therapy } \\
(n=36)\end{array}$ & $P$ \\
\hline Age $(\mathrm{months})^{\mathrm{c}}$ & $78 \pm 61$ & $70 \pm 50$ & $\mathrm{NS}^{*}$ \\
Weight $(\mathrm{kg})^{\mathrm{c}}$ & $25 \pm 18$ & $22 \pm 15$ & $\mathrm{NS}^{*}$ \\
Initial BP $(\mathrm{mmHg})^{\mathrm{c}}$ & & & \\
Systolic & $129 \pm 12$ & $135 \pm 14$ & $0.06^{* *}$ \\
Diastolic & $80 \pm 11$ & $83 \pm 13$ & $0.22^{* *}$ \\
Glomerulonephritis & $12(32 \%)$ & $6(17 \%)$ & $\mathrm{NS}^{* * * *}$ \\
Solid organ transplant & $15(40 \%)$ & $17(47 \%)$ & $\mathrm{NS}^{* * *}$ \\
\hline
\end{tabular}

* Mann-Whitney test, $* *$ two-tailed, unpaired $t$-test, $* * *$ Chi-square test

a Treatment courses in which BP control was achieved by isradipine alone

$\mathrm{b}$ Treatment courses in which additional agents were needed to achieve BP control

c Mean \pm standard deviation.

\section{Results}

Patients

A total of 80 children were identified as having been treated with isradipine at the University of Michigan between July 1995 and May 2000. However, BP data were missing for 4 patients, and were incomplete for another 4 patients. Two additional patients received isradipine on two separate occasions (before and after renal transplantation in both instances), leaving 72 patients and 74 treatment courses that are analyzed in this report. BP and other data from the 4 treatment courses in the 2 children who received isradipine twice were treated as coming from 4 separate patients for purposes of analysis.

Mean patient age was $74 \pm 55$ months $(6.2 \pm 4.6$ years), with a range of 1 week to 16.8 years. Of the 72 patients, $70(97.2 \%)$ had secondary hypertension. Underlying diagnoses for the 72 treatment courses in children with secondary hypertension included glomerulonephritis (18 patients), renal transplant (16 patients), liver transplant (16 patients), reflux nephropathy (5 patients), steroid-induced (5 patients), renovascular (3 patients), and other (9 patients). Hypertension in the solid organ transplant recipients was typically related to volume overload, the effects of immunosuppressive medications (corticosteroids and calcineurin inhibitors), pre-transplant hypertension, or a combination of these factors.

Of the 74 isradipine treatment courses, 68 (91.9\%) were begun during inpatient hospitalizations; the remaining 6 were begun in the outpatient department. In 46 of 74 treatment courses $(62 \%)$, isradipine was the initial antihypertensive agent utilized; in the remaining 28 instances, the patients were being treated with other agents and had isradipine added to their antihypertensive regimen.

In 38 of the 74 treatment courses $(51.4 \%)$, target BP was reached with isradipine alone. In 28 instances $(37.8 \%)$ one additional medication was required, and in 8 $(10.8 \%)$ two additional medications were needed. Supplemental medications utilized included diuretics (20 patients), beta-adrenergic antagonists (10 patients), direct vasodilators (minoxidil or hydralazine, 7 patients), angiotensin converting enzyme inhibitors (5 patients), and transdermal clonidine ( 2 patients). Pre-treatment charac-
Table 2 Isradipine dose received according to age, diagnosis, or dosing regimen (t.i.d. three times a day, q.i.d. four times a day)

\begin{tabular}{lcl}
\hline Group & $n$ & $\begin{array}{l}\text { Dose received } \\
(\mathrm{mg} / \mathrm{kg} \text { per day })\end{array}$ \\
\hline All treatment courses combined & 74 & $0.36 \pm 0.17$ \\
Age groups & & \\
0-1 year & 9 & $0.27 \pm 0.11$ \\
1-6 years & 32 & $0.38 \pm 0.20$ \\
6-12 years & 21 & $0.39 \pm 0.16$ \\
>12 years & 12 & $0.28 \pm 0.16$ \\
Solid organ transplant & 32 & $0.34 \pm 0.13$ \\
Glomerulonephritis & 18 & $0.38 \pm 0.16$ \\
Isradipine monotherapy & 38 & $0.31 \pm 0.12^{*}$ \\
Combination therapy & 36 & $0.40 \pm 0.21^{*}$ \\
t.i.d. dosing & 45 & $0.33 \pm 0.16^{* *}$ \\
q.i.d. dosing & 20 & $0.45 \pm 0.18^{* *}$ \\
\hline
\end{tabular}

$* P=0.04$ for comparison of monotherapy with combination therapy (two-tailed $t$-test), $* * P=0.01$ for comparison of t.i.d. dosing with q.i.d. dosing (two-tailed $t$-test)

teristics of the children requiring additional medications compared with those who did not require additional medications are listed in Table 1. Patient age, weight, and diastolic BP were similar in the two groups, where-as initial systolic BP tended to be higher in the group that required additional medications. There was no unusual distribution of diagnoses between the two groups (Table 1).

In 46 of the 74 treatment courses $(62 \%)$, a locally compounded isradipine suspension was used. As would be expected, these children were significantly younger than children who received isradipine capsules or extended-release tablets (mean age of patients receiving suspension was $39 \pm 28$ months vs. $132 \pm 36$ months for those receiving capsules or tablets, $P<0.0001)$.

\section{Isradipine dosing}

The mean isradipine dose received by all patients combined once target BP was reached was $0.36 \pm 0.17 \mathrm{mg} / \mathrm{kg}$ per day (range $0.07-0.9 \mathrm{mg} / \mathrm{kg}$ per day). Doses received by patients in specific age, diagnosis, and dosing groups are summarized in Table 2 . Linear regression analysis re- 
Table 3 BP reduction with isradipine treatment ${ }^{\mathrm{a}}$

* Two-tailed, paired $t$-test a For each row, the upper value is systolic $\mathrm{BP}($ mean $\pm \mathrm{SD})$ and the lower value is diastolic BP (all in $\mathrm{mmHg}$ )

Table 4 BP reduction with isradipine monotherapy ${ }^{\mathrm{a}}$

* Two-tailed, paired $t$-test

a For each row, the upper value is systolic $\mathrm{BP}($ mean $\pm \mathrm{SD})$ and the lower value is diastolic BP (all in $\mathrm{mmHg}$ )

\begin{tabular}{lccccc}
\hline Group & $n$ & $\begin{array}{l}\text { BP prior to } \\
\text { isradipine treatment }\end{array}$ & $\begin{array}{l}\text { BP during } \\
\text { isradipine treatment }\end{array}$ & $\begin{array}{l}\text { Mean change } \\
\text { in BP }\end{array}$ & $\mathrm{P}^{*}$ \\
\hline Age 0-1 years & 9 & $120 \pm 12$ & $100 \pm 15$ & $21 \pm 12$ & 0.001 \\
& & $77 \pm 15$ & $56 \pm 12$ & $21 \pm 15$ & 0.003 \\
Age 1-6 years & 32 & $131 \pm 9$ & $116 \pm 9$ & $15 \pm 12$ & $<0.0001$ \\
& & $80 \pm 12$ & $69 \pm 8$ & $12 \pm 15$ & $<0.0001$ \\
Age 7-12 years & 21 & $133 \pm 14$ & $122 \pm 10$ & $11 \pm 13$ & 0.001 \\
& & $81 \pm 9$ & $70 \pm 10$ & $12 \pm 12$ & $<0.0001$ \\
Age >12 years & 12 & $141 \pm 17$ & $127 \pm 10$ & $13 \pm 14$ & 0.01 \\
& & $88 \pm 14$ & $76+9$ & $12 \pm 18$ & 0.04 \\
All treatment courses & 74 & $132 \pm 13$ & $117 \pm 13$ & $14 \pm 13$ & $<0.0001$ \\
combined & & $81 \pm 12$ & $68 \pm 11$ & $13 \pm 15$ & $<0.0001$ \\
\hline
\end{tabular}

\begin{tabular}{lccccc}
\hline Group & $n$ & $\begin{array}{l}\text { BP prior to } \\
\text { isradipine treatment }\end{array}$ & $\begin{array}{l}\text { BP during } \\
\text { isradipine treatment }\end{array}$ & $\begin{array}{l}\text { Mean change } \\
\text { in BP }\end{array}$ & P* \\
\hline Age 0-1 years & 6 & $117 \pm 13$ & $97 \pm 17$ & $21 \pm 11$ & 0.006 \\
& 14 & $132 \pm 13$ & $58 \pm 15$ & $19 \pm 12$ & 0.01 \\
Age 1-6 years & & $79 \pm 13$ & $117 \pm 8$ & $16 \pm 13$ & 0.001 \\
& 10 & $127 \pm 11$ & $70 \pm 10$ & $9 \pm 18$ & 0.08 \\
Age 7-12 years & & $79 \pm 8$ & $120 \pm 10$ & $7 \pm 11$ & 0.07 \\
& 8 & $134 \pm 13$ & $71 \pm 8$ & $8 \pm 11$ & 0.04 \\
Age >12 years & & $84 \pm 8$ & $124 \pm 10$ & $10 \pm 11$ & 0.03 \\
& 38 & $129 \pm 12$ & $75+8$ & $9 \pm 6$ & 0.003 \\
All treatment courses & $80 \pm 11$ & $116 \pm 14$ & $13 \pm 12$ & $<0.0001$ \\
combined & & $80 \pm \pm 11$ & $10 \pm 13$ & $<0.0001$ \\
\hline
\end{tabular}

vealed no significant association between patient age and isradipine dose. Patients whose BP was controlled with isradipine alone received a significantly lower dose than those in whom BP control required isradipine in combination with other agents (Table 2). Dose was similar in children who received isradipine as a suspension compared with those who received capsules or tablets $(0.36 \pm 0.18 \mathrm{mg} / \mathrm{kg}$ per day vs. $0.34 \pm 16 \mathrm{mg} / \mathrm{kg}$ per day, $P=\mathrm{NS}$ ).

Isradipine was administered three times daily in 45 instances $(60.8 \%)$, four times daily in 20 instances $(27 \%)$, and once or twice daily in the remaining 9 instances $(12.2 \%)$. The mean isradipine dose when it was administered four times daily was significantly higher than when it was given three times daily (Table 2). There was no relationship seen between patient age and dosing frequency. However, children who received isradipine suspension tended to be more likely to receive it more than twice a day than those who received isradipine capsules: $93 \%$ of suspension recipients were dosed three or four times daily versus $79 \%$ of capsule recipients $(P=0.07$, Fisher's exact test $)$.

\section{Effects of isradipine treatment}

BP data for all 74 treatment courses and for specific age groups are summarized in Table 3. The mean time between the baseline (pre-isradipine treatment) and follow-up BP was $8 \pm 9$ days (range $1-42$ days). Isradipine treatment pro- duced a significant reduction in both systolic and diastolic BP in all age groups. There was no significant change in heart rate with isradipine treatment, except in infants $<1$ year old, in whom heart rate decreased from 148 to 132 beats per min $(P=0.04$, two-tailed paired $t$-test $)$.

For further assessment of the effects of isradipine treatment, the BP responses in the 38 treatment courses when BP control was achieved by isradipine alone were examined separately. Results are summarized in Table 4. In general, for almost every age group, there was a significant reduction in BP following isradipine treatment. There was no significant change in heart rate with isradipine treatment for any age group (data not shown).

To determine whether there was a dose-response relationship between isradipine dose and the change in BP, linear regression analysis was performed. In order to eliminate the effects of other antihypertensive medications, this analysis was restricted to the 38 treatment courses when isradipine was used as monotherapy. No significant relationship was demonstrated between isradipine dose (in $\mathrm{mg} / \mathrm{kg}$ per day) and change in systolic or diastolic BP, expressed either as absolute change in $\mathrm{mmHg}$ (baseline BP minus treatment $\mathrm{BP}$ ), or as percentage change $[($ baseline $\mathrm{BP}$ minus treatment $\mathrm{BP}) /$ baseline $\mathrm{BP}]$.

\section{Adverse effects}

Adverse effects attributed to isradipine treatment occurred in 7 of 74 treatment courses $(9.5 \%)$ during the pe- 
riod of follow-up in this study, for a rate of 0.35 adverse events per patient-month of treatment. Adverse effects reported included headache ( 2 patients), flushing ( 2 patients), dizziness (1 patient), tachycardia (1 patient), and unspecified (1 patient). No patient reported more than one adverse effect. There was no difference in the incidence of adverse effects between children treated with isradipine alone compared with those treated with isradipine plus additional medications, and no difference in the dose of isradipine received between children with and without adverse effects.

\section{Discussion}

In this single-center study, the second-generation dihydropyridine calcium antagonist isradipine was administered to a relatively large group of hypertensive children that are typical of patients with hypertension seen at pediatric nephrology centers. In these children, nearly all of whom had secondary hypertension and were being treated as hospital inpatients, isradipine significantly lowered both systolic and diastolic BP, an effect that was present no matter whether isradipine was being administered alone or in combination with other antihypertensive agents. There was no significant effect of isradipine on heart rate, except in infants $<1$ year of age who received isradipine in combination with other agents. The lack of an effect on heart rate in patients of all ages receiving isradipine monotherapy suggests that this change may have been related to the additional antihypertensives received by some of those infants. Use of a locally compounded isradipine suspension facilitated treatment of children as young as 1 week of age. Although the incidence of adverse effects related to isradipine treatment was not insignificant, it was smaller than that reported by other investigators [15] and compared favorably with our experience with the calcium antagonist amlodipine [13].

Several findings in this study merit further discussion. As has been found by other investigators $[15,16]$, the doses of isradipine administered to the children in this study were significantly greater than doses typically used in adults. Although this has also been reported for several other calcium antagonists, most notably amlodipine [13], because of the retrospective, uncontrolled design of this study it is difficult to know whether children truly require greater doses than adults or whether this finding simply represents the prescribing practices at our center. It may also be related to the fact that most of the children received isradipine in suspension form. Although the suspension remains stable after compounding [12], there may be differences in bioavailability of the suspension compared with the commercially available formulations that may reduce the magnitude of the antihypertensive effect of the suspension.

Another important finding was that most of the children in this study seemed to require administration of isradipine three or four times daily to maintain stable BP. While this is greater than the recommended frequency for adults of two times per day, it is consistent with the relatively short elimination half-life of isradipine of as little as $6 \mathrm{~h}$ [10]. It may also be related to the extensive first-pass hepatic metabolism of isradipine [10] and the possibility that children may clear this drug more rapidly than adults due to their proportionately greater hepatic mass. Clearly, a formal pharmacokinetic study would be necessary to settle this issue.

Despite the wide range of doses of isradipine received by the patients in this study, and despite the fact that many children received proportionately higher doses than those recommended for adults, we were unable to demonstrate a dose-response relationship between the dose of isradipine and the magnitude of BP reduction. This is most likely a function of the retrospective study design and also of the relatively small number of patients included compared with clinical trials of other antihypertensives. This question, as well as the pharmacokinetic issues, could be answered by a multi-center prospective trial similar to those that have been conducted for other antihypertensives under the auspices of the Food and Drug Modernization Act of 1997 [17].

Pediatric use of isradipine has previously been reported $[15,16,18]$. Johnson et al. [16] studied the effects of isradipine in the management of 53 hospitalized children with hypertension, and found that isradipine produced a mean reduction in BP of $12 \% \pm 13 \%$ for systolic pressure and $17 \% \pm 20 \%$ for diastolic pressure. Doses received were much higher than those reported effective in adults, especially in children with renal disease. No data were reported regarding the incidence of adverse effects. Similarly, Strauser et al. [18] reported that isradipine effectively lowered BP in 10 of 12 children, most of whom had renal causes of hypertension. No information was included in their report regarding the magnitude of BP reduction produced by isradipine, nor the incidence of adverse effects.

Limitations of this study have been alluded to above and include the retrospective design, lack of a control group, and possible selection bias. However, it should be stressed that the relatively large number of children included compared with other single-center studies of antihypertensive agents in children makes it likely that our conclusions regarding isradipine in this patient population are correct. In addition, we believe that the efforts that were made to identify children treated with isradipine beyond those with which the investigators were personally familiar makes it unlikely that the study findings are significantly affected by selection bias. Finally, we should point out that since most of the children in this study had secondary hypertension, we cannot draw any conclusions about the use of isradipine in children with primary hypertension.

The ideal agent for treatment of children with hypertension remains unknown. We believe that isradipine represents a useful option, particularly in children like those in this report who have secondary hypertension requiring prompt treatment. We are hopeful that a prospective, randomized controlled study will be performed to more fully evaluate the role of isradipine in the management of pediatric hypertension. 
Acknowledgements Presented in part at the 33rd Annual Meeting of the American Society of Nephrology, Toronto, Ontario, October 2000.

\section{References}

1. Flynn JT (2001) Pediatric use of antihypertensive medications: much more to learn. Curr Ther Res Clin Exp 62:314-328

2. National High Blood Pressure Education Program Working Group (1996) Update on the 1987 Task Force Report on High Blood Pressure in Children and Adolescents: a working group report from the National High Blood Pressure Education Program. Pediatrics 98:649-658

3. Nahata MC (1999) Lack of pediatric drug formulations. Pediatrics 104:607-609

4. Strife CF, Quinlan M, Waldo FB, Fryer CJ, Jackson EC, Welch TR, McEnery PT, West CD (1986) Minoxidil for control of acute blood pressure elevation in chronically hypertensive children. Pediatrics 78:861-865

5. Alexander KS, Pudipeddi M, Parker GA (1993) Stability of hydralazine hydrochloride syrup compounded from tablets. Am J Hosp Pharm 50:683-686

6. Blowey DL (2001) Antihypertensive agents: mechanisms of action, safety profiles, and current uses in children. Curr Ther Res Clin Exp 62:298-313

7. Flynn JT, Pasko DA (2000) Calcium channel blockers: pharmacology and place in therapy of pediatric hypertension. Pediatr Nephrol 15:302-316

8. Griebenow R, Kaufmann W, Krämer L, Steffen HM, Wambach G, Burger KJ, Welzel D (1990) Isradipine: a new calcium antagonist with strong vasodilatory but negligible cardiodepressive effects. J Cardiovasc Pharmacol 15 [Suppl 1]:S84-S86
9. Saragoça MA, Portela JE, Plavnik F, Ventura RP, Lotaif L, Ramos OL (1992) Isradipine in the treatment of hypertensive crisis in ambulatory patients. J Cardiovasc Pharmacol 19 [Suppl 3]:S76-S78

10. Brogden RN, Sorkin EM (1995) Isradipine: an update of its pharmacodynamic and pharmacokinetic properties and therapeutic efficacy in the treatment of mild to moderate hypertension. Drugs 49:618-649

11. Kirch W, Burger KJ, Weidinger G, Welzel D (1990) Efficacy and tolerability of the new calcium antagonist isradipine in essential hypertension. J Cardiovasc Pharmacol 15 [Suppl 1]: S55-S59

12. MacDonald JL, Johnson CE, Jacobson P (1994) Stability of isradipine in an extemporaneously compounded oral liquid. Am J Hosp Pharm 51:2409-2411

13. Flynn JT, Smoyer WE, Bunchman TE (2000) Treatment of hypertensive children with amlodipine. Am J Hypertens 13:1061-1066

14. National High Blood Pressure Education Program Task Force on Blood Pressure Control in Children (1987) Report of the Second Task Force on Blood Pressure Control in Children 1987. Pediatrics 79:1-25

15. Wells TG, House M, Belsha CW, Berry PL (1995) Isradipine for treatment of hypertension in children (abstract). J Invest Med 43:59A

16. Johnson CE, Jacobson PA, Song MH (1997) Isradipine therapy in hypertensive pediatric patients. Ann Pharmacother 31:704-707

17. Wells TG (1999) Trials of antihypertensive therapies in children. Blood Press Monit 4:189-192

18. Strauser LM, Groshong T, Tobias JD (2000) Initial experience with isradipine for the treatment of hypertension in children. South Med J 93:287-293 